\title{
Technè
}

La science au service de l'histoire de l'art et de la préservation des biens culturels

42 | 2015

Science et conservation

\section{Du nouveau sur Della Robbia à Marseille : enquête historique et scientifique}

New findings about the Della Robbias in Marseille: a historical and scientific investigation

\section{Axelle Davadie, Marc Bormand, Anne Bouquillon, Christel Doublet,} Élisabeth Mognetti et Solange Rizoulières

\section{(2) OpenEdition}

Journals

Édition électronique

URL : http://journals.openedition.org/techne/6897

DOI : 10.4000/techne.6897

ISSN : 2534-5168

Éditeur

C2RMF

\section{Édition imprimée}

Date de publication : 1 décembre 2015

Pagination : 62-71

ISBN : 978-2-7118-6249-8

ISSN : 1254-7867

Référence électronique

Axelle Davadie, Marc Bormand, Anne Bouquillon, Christel Doublet, Élisabeth Mognetti et Solange

Rizoulières, « Du nouveau sur Della Robbia à Marseille : enquête historique et scientifique », Technè [En ligne], 42 | 2015, mis en ligne le 01 décembre 2015, consulté le 11 mars 2021. URL : http:// journals.openedition.org/techne/6897 ; DOI : https://doi.org/10.4000/techne.6897

La revue Technè. La science au service de l'histoire de l'art et de la préservation des biens culturels est mise à disposition selon les termes de la Licence Creative Commons Attribution - Pas d'Utilisation Commerciale - Pas de Modification 4.0 International. 


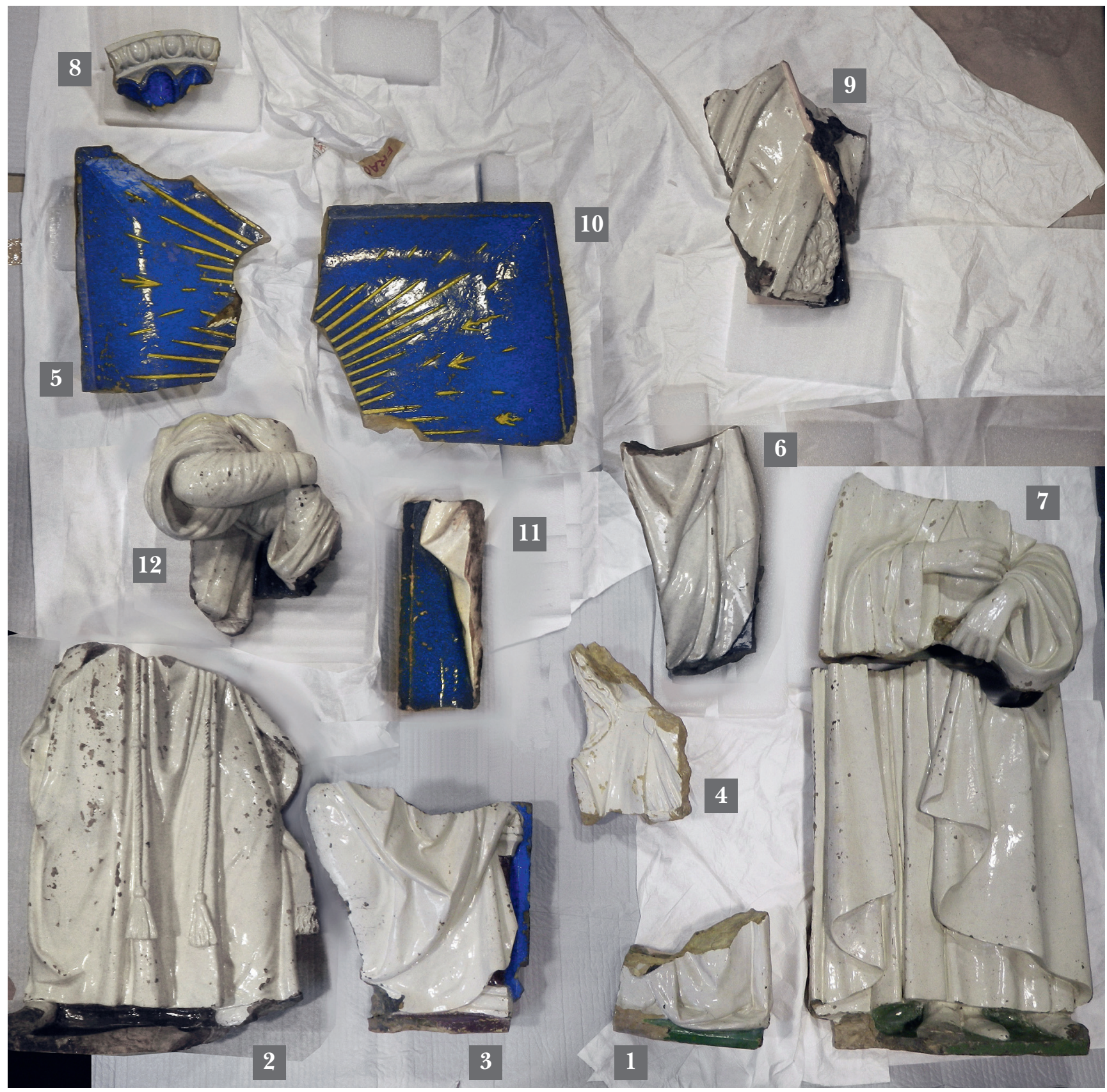

Fig. 1. État et vue d'ensemble des fragments robbiesques. @ C2RMF/Axelle Davadie. 
Axelle Davadie

Marc Bormand

Anne Bouquillon

Christel Doublet

Élisabeth Mognetti

Solange Rizoulières

\section{Du nouveau sur Della Robbia à Marseille : enquête historique et scientifique}

Résumé. La rénovation du musée d'Histoire de Marseille a permis l'étude historique et scientifique de fragments de céramique glaçurée et confirmé leur attribution ancienne aux Della Robbia. Elle a ouvert la piste de leur provenance, vraisemblablement une chapelle florentine dans l'église de l'Observance. L'iconographie est plus délicate à définir et leur présentation future s'en ressent.

Mots-clés. Terre cuite, Della Robbia, glaçure, Observance, Marseille, Renaissance, fragments, fä̈ence, iconographie.
New findings about the Della Robbias in Marseille: a historical and scientific investigation

\begin{abstract}
The renovation of the History Museum in Marseille made it possible to carry out a historical and scientific study of fragments of glazed ceramics and confirm their earlier attribution to the Della Robbias. Their provenance was traced in all likelihood to a Florentine chapel in the church of the Observance. Defining the iconography is a more delicate matter, and it shows in the future display of the fragments.
\end{abstract}

Keywords. Terracotta, Della Robbia, glaze, Observance, Marseille, Renaissance, fragments, faience, iconography.
L'obtention du titre de «capitale européenne de la culture » par Marseille en 2013 a permis la réalisation de grands projets culturels, en particulier la rénovation et l'extension du musée d'Histoire (MHM). Les restaurations nécessitées par le nouveau parcours ont concerné un millier de pièces, dont un ensemble de douze fragments de terre cuite glaçurée, considérés de longue date comme étant des productions de l'atelier des Della Robbia. La ville de Marseille conserve déjà des pièces importantes de cette prestigieuse dynastie de sculpteurs florentins de la Renaissance, parmi lesquelles la Mise au tombeau de la Vieille Major, qui n'est malheureusement plus exposée depuis sa restauration en $1998^{1}$. Redécouvrir aujourd'hui ces fragments, tenter d'apporter des éléments de réponse aux affirmations récurrentes d'une production robbiesque marseillaise et surtout redonner sens, histoire et visibilité à ces témoignages uniques, voilà ce qui sera le fil conducteur de l'article. Tout au long de cette recherche complexe, mettant en commun les données de la matière, les informations éparses dans les archives, celles des mémoires multiples, nous ferons nôtre cette phrase de Pierre Trabaud (1883) : "Après tout, il reste encore, pour ceux qui se passionnent en faveur d'un passé émouvant, la tâche ambitieuse de pénétrer les secrets des origines, avec la douce espérance que l'avenir révèle quelque donnée meilleure, propre à parfaire des études fatalement incomplètes. »

\section{Présentation des fragments}

Les douze fragments (fig. 1, Tableau I) appartiennent aux collections actuelles du musée d'Histoire de Marseille et, pour certains, aux objets du premier musée d'archéologie installé au château Borély en 1863. Ces pièces n'avaient pas été inventoriées en totalité jusqu'à présent ${ }^{2}$. Seules trois (fr. $7,2,12$ ) sont mentionnées dans l'inventaire de 1895 (fig. 2 et 3 ) réalisé par Michel Clerc ${ }^{3}$ comme situées «dans le cabinet faisant suite à la salle de la céramique moderne ». De plus, pour les seuls 22 et 23, il précise «Don de Mr H. Augier ${ }^{4}$ ” qui a travaillé au musée de 1864 jusqu'à sa mort en 1889. Il a beaucoup acheté, aussi bien des objets issus de fouilles marseillaises que d'autres qu'il a vendus ou donnés au musée.

Déjà en 1883, Pierre Trabaud, membre de l'Académie de Marseille, publie plusieurs articles consacrés aux ouvres des Della Robbia dans sa ville ${ }^{5}$. Il évoque bien entendu la Mise au Tombeau de l'ancienne cathédrale de la Major et le Christ en croix vêtu d'une robe, autrefois dans l'église des Accoules ${ }^{6}$, mais mentionne également des « débris recueillis au château Borély et selon la tradition dans les vieux quartiers de la cité phocéenne ». Il décrit « les personnages décapités portant la robe des moines ayant la corde à la ceinture » sans en donner le nombre (il pourrait s'agir des fr. 2 et 7). Ils lui semblent appartenir «à l'ordre des Observantins » mais celui qu'il reproduit (fr. 7) n'a aucune corde à la ceinture. Le « corsage

Axelle Davadie, conservateur, C2RMF (axelle.davadie@culture.gouv.fr). Marc Bormand, conservateur en chef, musée du Louvre, département des Sculptures (Marc.Bormand@louvre.fr).Anne Bouquillon, ingénieur de recherche, C2RMF (anne.bouquillon@culture.gouv.fr).

Christel Doublet, ingénieur d'étude, C2RMF (christel.doublet@culture.gouv.fr). Élisabeth Mognetti, conservateur général honoraire (elisabeth.mognetti@bbox.fr). Solange Rizoulières, conservateur, musée d’Histoire de Marseille (srizoulieres@mairie-marseille.fr). 
Tableau I. Liste des fragments (H. = hauteur en mètres et 1. = largeur en mètres)

\begin{tabular}{|c|c|c|c|}
\hline Inv. 2014.0.1 à 2014.0.12 & Couleur de la terrasse & Description & Inventaire 1895 \\
\hline $\begin{array}{l}\text { Fragment } 1 \\
\text { H. } \times 1 .: 0,22 \times 0,24\end{array}$ & Terrasse verte & $\begin{array}{l}\text { Bas de vêtement d'un personnage } \\
\text { agenouillé ? }\end{array}$ & \\
\hline $\begin{array}{l}\text { Fragment } 2 \\
\text { H. } \times 1 .: 0,63 \times 0,44\end{array}$ & Terrasse parme & $\begin{array}{l}\text { Partie inférieure du corps d'un } \\
\text { personnage, genoux légèrement } \\
\text { repliés, pied droit écarté dans un } \\
\text { mouvement dynamique vers senestre }\end{array}$ & $\begin{array}{l}\mathrm{N}^{\circ} 23 \text { - Fragment de statue en } \\
\text { faïence, plus incomplète que la } \\
\text { précédente et représentant aussi un } \\
\text { ecclésiastique : il n’y a que le bas du } \\
\text { corps à partir des cuisses. } \\
\text { H. x } 1 .: 0,63 \text { x } 0,44\end{array}$ \\
\hline $\begin{array}{l}\text { Fragment } 3 \\
\text { H. x } 1 .: 0,40 \times 0,33\end{array}$ & $\begin{array}{l}\text { Terrasse parme } \\
\text { mouchetée de blanc }\end{array}$ & $\begin{array}{l}\text { Drapé du vêtement d'un personnage } \\
\text { assis sur un siège mouluré, sur fond } \\
\text { bleu, placé dans un angle inférieur } \\
\text { senestre }\end{array}$ & \\
\hline $\begin{array}{l}\text { Fragment } 4 \\
\text { H. } \times 1 .: 0,31 \times 0,20\end{array}$ & & $\begin{array}{l}\text { Partie de torse et de visage féminins, } \\
\text { presque de face, tête inclinée à dextre, } \\
\text { trace d'arrachement au niveau du } \\
\text { fermail du manteau, amorce de drapé } \\
\text { recouvrant le manteau sur l'épaule } \\
\text { droite }\end{array}$ & \\
\hline $\begin{array}{l}\text { Fragment } \mathbf{5} \\
\text { H. } \times 1 .: 0,42 \times 0,22\end{array}$ & & $\begin{array}{l}\text { Angle supérieur aux bords incurvés } \\
\text { sur fond bleu à rayons d'or émanant } \\
\text { de personnes divines (?), montrant } \\
\text { le reste d'une aile et, en négatif, } \\
\text { la présence d'une tête de chérubin }\end{array}$ & \\
\hline $\begin{array}{l}\text { Fragment } 6 \\
\text { H. } \times 1: 0,39 \times 0,17\end{array}$ & Amorce de terrasse verte & $\begin{array}{l}\text { Drapé retombant verticalement, } \\
\text { sur fond bleu }\end{array}$ & \\
\hline $\begin{array}{l}\text { Fragment } 7 \text { (en } 2 \text { parties) } \\
\text { H. x 1. : } 1,03 \times 0,45\end{array}$ & Terrasse verte & $\begin{array}{l}\text { Ecclésiastique ou religieux tenant de } \\
\text { la main gauche la base d'un objet dans } \\
\text { le repli du manteau et, dans la main } \\
\text { droite, la partie supérieure du même } \\
\text { objet ayant laissé une trace de jaune au- } \\
\text { dessus des doigts (une croix dorée ?), } \\
\text { le pied d'un personnage situé derrière } \\
\text { lui est visible sur la terrasse }\end{array}$ & $\begin{array}{l}\mathrm{N}^{\circ} 22 \text { - Fragment de statue } \\
\text { en faience représentant un } \\
\text { ecclésiastique, les deux mains y sont } \\
\text { mais le haut du corps manque } \\
\text { à partir de l'estomac. } \\
\text { H. } x 1 .: 1 \times 0,45\end{array}$ \\
\hline $\begin{array}{l}\text { Fragment } 8 \\
\text { H. } \times 1 .: 0,13 \times 0,21\end{array}$ & & $\begin{array}{l}\text { Fragment décoratif arrondi à motif de } \\
\text { coquille bordée d'oves }\end{array}$ & \\
\hline $\begin{array}{l}\text { Fragment } 9 \\
\text { H. } \times 1 .: 0,25 \times 0,12\end{array}$ & & $\begin{array}{l}\text { Sur fond bleu, bas du corps, de } \\
\text { la ceinture aux genoux, de saint } \\
\text { Jean-Baptiste tenant un bâton doré } \\
\text { (jaune) avec trace d'arrachement de } \\
\text { sa main droite sur le bâton (et d'un } \\
\text { phylactère?) }\end{array}$ & \\
\hline $\begin{array}{l}\text { Fragment } 10 \\
\text { H. } \times 1 .: 0,40 \times 0,43\end{array}$ & & $\begin{array}{l}\text { Angle supérieur aux bords incurvés sur } \\
\text { fond bleu à rayons d'or émanant de } \\
\text { personnes divines (?) }\end{array}$ & \\
\hline $\begin{array}{l}\text { Fragment } 11 \\
\text { H. x } 1 .: 0,33 \times 0,11\end{array}$ & & $\begin{array}{l}\text { Pli de vêtement sur fond bleu, bord } \\
\text { dextre incurvé }\end{array}$ & \\
\hline $\begin{array}{l}\text { Fragment } 12 \\
\text { H. x 1. : } 0,26 \times 0,27\end{array}$ & & $\begin{array}{l}\text { Torse d'ange, les bras croisés sur la } \\
\text { poitrine, légèrement incliné et tourné } \\
\text { vers senestre, sur fond bleu }\end{array}$ & $\begin{array}{l}\mathrm{N}^{\circ} 24 \text { - Débris de statue en faïence. } \\
\text { Personnage ayant les bras croisés sur } \\
\text { l'estomac = il n'existe que les bras. } \\
\text { Tout le reste a disparu. } \\
\text { 1. : } 0,29\end{array}$ \\
\hline
\end{tabular}




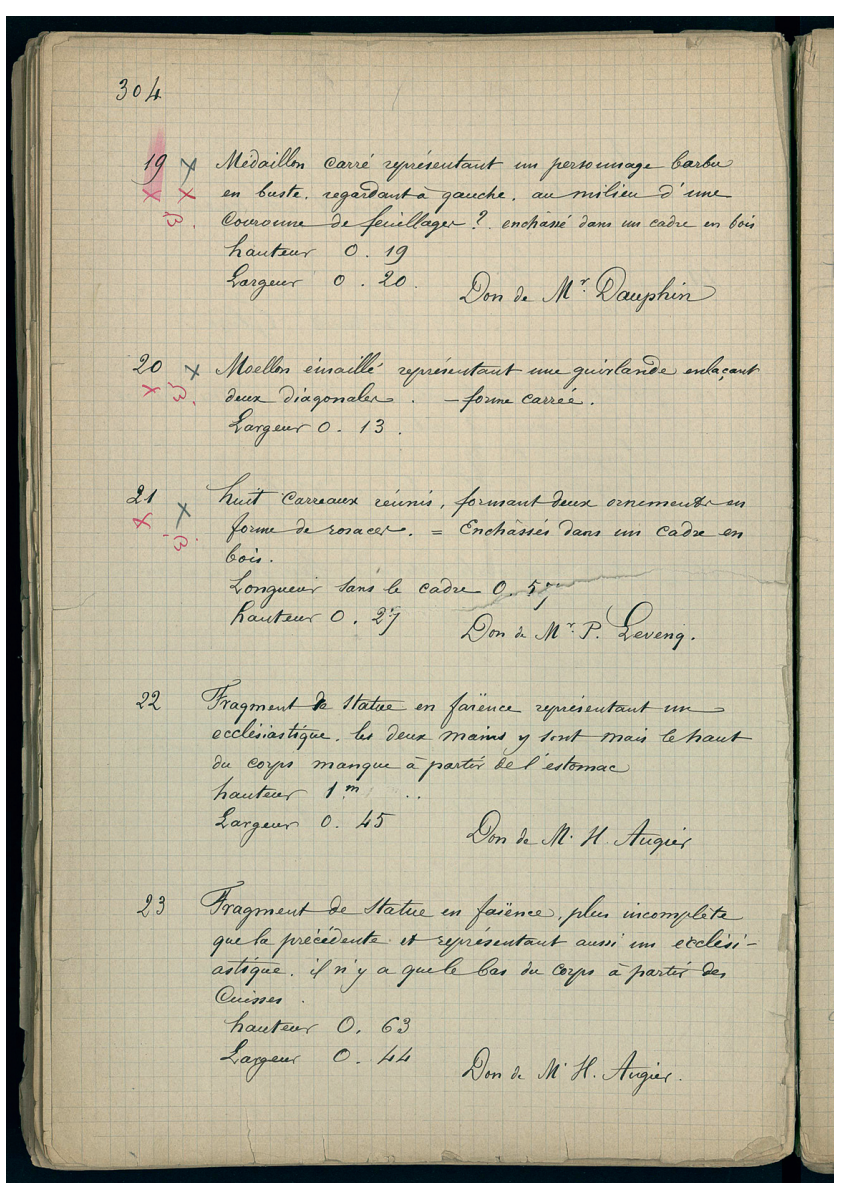

Fig. 2. La page 304 de l'inventaire du musée Borély mentionne les deux personnages les mieux conservés et en donne les dimensions. (c) Musée d'Histoire de Marseille.

gracieux d'une femme avec les bras croisés (fr. 12), les fragments bleus enrichis de rayons d'or ${ }^{7}{ }($ fr. 5 et 10$)$ en faisaient partie.

On peut s'étonner dès lors que l'inventaire de 1895 ne tienne pas compte de la publication de 1883 . On pense maintenant que les cinq morceaux décrits sont entrés dans les collections entre 1864 et 1882, date à laquelle Trabaud les présente lors d'une conférence. Pour les sept autres fragments, on ne sait pas s'ils sont arrivés au même moment ou s'il s'agit d'entrées postérieures, au gré des découvertes.

\section{Origine des œuvres : Marseille ou Toscane?}

Pierre Trabaud, comme ses prédécesseurs, s'intéresse surtout à ces œuvres en ce qu'elles attesteraient une origine robbiesque de la faïence produite à Marseille. Il en a envoyé un fragment ${ }^{8}$ au directeur d'une fabrique florentine, qui lui a certifié qu'il s'agissait bien, pour la pâte comme pour l'émail, d'une technique identique aux travaux des Della Robbia. Il lui paraît dès lors peu vraisemblable que ceux-ci aient importé de l'argile de Toscane pour une cuisson dans des fours marseillais.

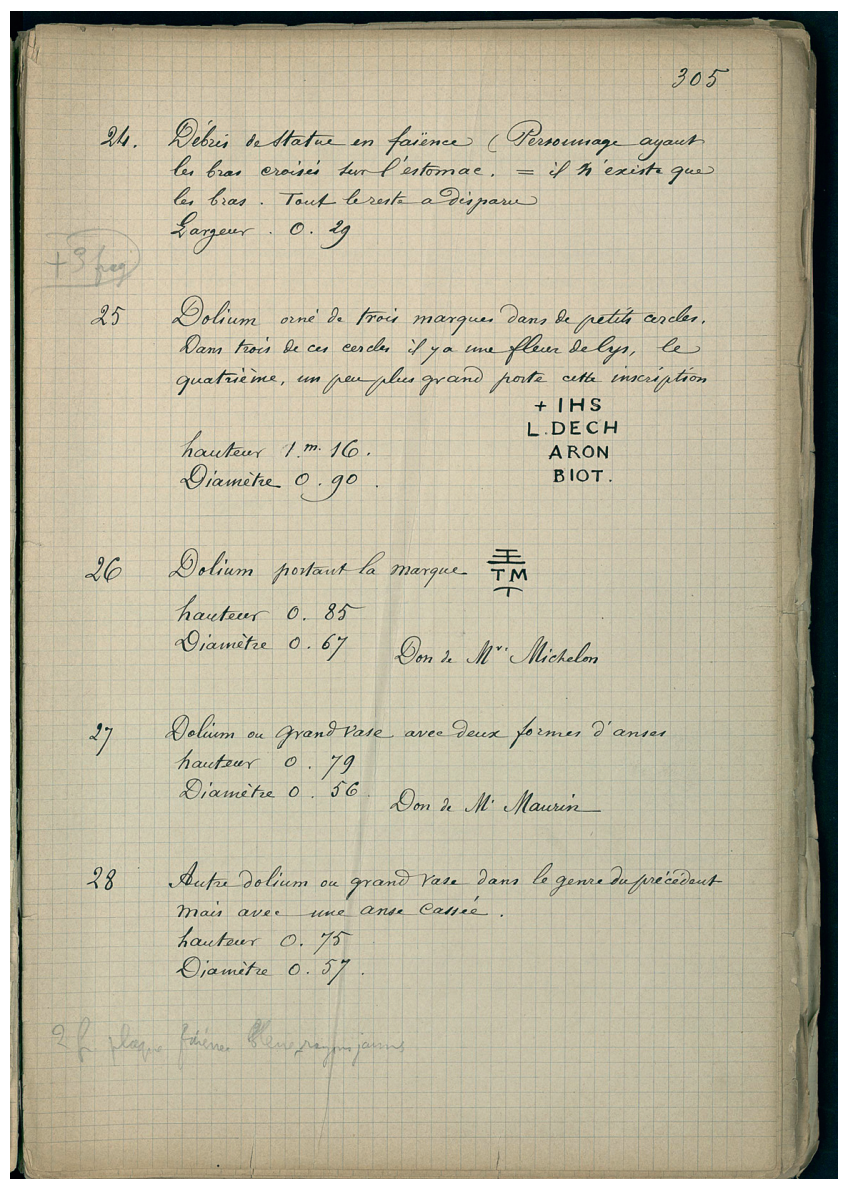

Fig. 3. La page 305 de l'inventaire du musée Borély enregistre le troisième fragment, personnage aux bras croisés. (C) Musée d'Histoire de Marseille.

Il envisage donc plutôt une importation d'Italie. Il a par ailleurs bien remarqué, sur le retable de la Major, la découpe des personnages visant non seulement à permettre la cuisson, mais aussi à en faciliter le transport.

À cet égard, les pressentiments de Pierre Trabaud étaient justes et sont devenus, aujourd'hui, des certitudes grâce aux analyses physico-chimiques réalisées sur l'ensemble des douze éléments. En effet, nous avons pu prélever quelques milligrammes de la pâte constitutive sur chacun et les analyser par PIXE selon les protocoles décrits dans Bouquillon $e t$ al. (2010). La composition chimique élémentaire des pâtes des terres cuites robbiesques est bien connue grâce à l'étude de plus de quatre-vingts œuvres réparties dans divers musées, en Italie et en France. Elle est particulièrement stable pendant toute la période d'activité de la bottega des Della Robbia à Florence du fait d'un approvisionnement constant dans une carrière d'argile achetée par Luca della Robbia dans le Val d'Arno. Le tableau ci-dessous en livre les caractéristiques essentielles.

Nous avons retrouvé des compositions tout à fait similaires pour tous les fragments, quelle que soit la couleur de la pâte, ivoire ou plus rosée (voir Tableau II). Il s'agit donc bien d'une 
Tableau II. Composition chimique élémentaire moyenne des pâtes robbiesques - Comparaison avec celles des fragments Données PIXE exprimées en \% en poids d'oxydes

\begin{tabular}{|l|l|l|l|l|l|l|l|l|l|l|l|l|}
\hline & $\mathbf{N a}_{\mathbf{2}} \mathbf{O}$ & $\mathbf{M g O}$ & $\mathbf{A l}_{\mathbf{2}} \mathbf{O}_{\mathbf{3}}$ & $\mathbf{S i O}_{\mathbf{2}}$ & $\mathbf{P}_{\mathbf{2}} \mathbf{O}_{\mathbf{5}}$ & $\mathbf{S O}_{\mathbf{3}}$ & $\mathbf{C l}$ & $\mathbf{K}_{\mathbf{2}} \mathbf{O}$ & $\mathbf{C a O}$ & $\mathbf{T i O}_{2}$ & $\mathbf{M n O}$ & $\mathbf{F e}_{2} \mathbf{O}_{\mathbf{3}}$ \\
\hline $\begin{array}{l}\text { Moyenne des fragments } \\
\text { Marseille }\end{array}$ & 1,87 & 3,14 & 12,80 & 50,49 & 0,21 & 1,37 & 0,81 & 1,98 & 22,03 & 0,55 & 0,13 & 4,63 \\
\hline Écart-type & 0,9 & 0,3 & 0,5 & 2,0 & 0,0 & 0,7 & 0,5 & 0,2 & 1,2 & 0,0 & 0,0 \\
\hline Moyenne des Robbiesques & 1,06 & 2,85 & 13,20 & 52,07 & 0,23 & $\mathrm{nd}$ & $\mathrm{nd}$ & 2,06 & 22,99 & $\mathrm{nd}$ & 0,11 & 4,9 \\
\hline Écart-type & 0,4 & 1,4 & 0,7 & 2,5 & 0,1 & $n d$ & $n d$ & 0,2 & 2,3 & $n d$ & 0,02 & 0,4 \\
\hline
\end{tabular}

production totalement compatible avec les œuvres robbiesques florentines.

L'étude des glaçures permet d'aller plus loin dans la collecte de données susceptibles d'orienter les recherches. Ces dernières ont été étudiées soit par analyse PIXE directement sur les objets, soit par microscopie électronique à balayage, sur prélèvement millimétrique inclus dans la résine et poli.

Luca et Andrea della Robbia, lorsqu'ils ont inventé l'art de la sculpture glaçurée, ont dû chercher les meilleures compositions des couvertes vitreuses afin d'obtenir le rendu parfait du blanc marmoréen et du bleu profond tout en assurant un indispensable accord entre pâte et glaçure, nécessaire à une excellente résistance dans le temps. Ici, les glaçures sont très homogènes, elles sont colorées sur toute l'épaisseur. Riches en plomb (30-40\% $\mathrm{PbO})$ et en silice $\left(20-45 \% \mathrm{SiO}_{2}\right)$, elles sont opacifiées par des microcristaux de cassitérite (15 à $20 \%$ $\mathrm{SnO}_{2}$ ), notamment pour le bleu et le blanc. Le vert des bases, souvent un peu plus hétérogène, est moins stannifère, de même que le jaune ; l'opacification est obtenue, pour ces couleurs, par la présence de microcristaux d'antimoniates de plomb, jouant le double rôle de colorant et d'opacifiant. Les agents colorants sont traditionnels : cuivre et antimoniates de plomb pour le vert clair, antimoniates de plomb pour le jaune, manganèse pour le violet, cobalt pour le bleu. Ainsi, les caractéristiques chimiques et microstructurales des glaçures permettent-elles également d'étayer l'hypothèse d'œuvres des Della Robbia. Mais d'autres critères matériels livrent une information chronologique plus précise : les fortes teneurs en étain (> $15 \% \mathrm{SnO}_{2}$ ) signent plutôt des œuvres précoces de Luca et de la première partie de l'activité d'Andrea ; ces teneurs ont semble-t-il tendance à diminuer pour les œuvres plus tardives ${ }^{9}$. Si l'on se focalise maintenant sur les glaçures bleues, on observe que seul le fragment à oves (fr. 8) se distingue par la présence d'arsenic (As) lié au cobalt (Co), alors que, pour les autres, l'arsenic est absent et le cobalt est lié au fer $(\mathrm{Fe})$ et au nickel (Ni). L'association Co-As est typique d'œuvres postérieures à 1520, tandis que l'association Co-Ni-Fe est spécifique des œuvres antérieures à cette date ${ }^{10}$.
À l'issue des analyses des matériaux constitutifs, on peut donc confirmer que ces fragments appartiennent à une production florentine, mais qu'il y a probablement plusieurs œuvres dont l'une, à laquelle appartient le fragment à oves, a été créée après 1520.

La diffusion de reliefs des Della Robbia vers Marseille et plus largement vers le Sud de la France est bien connue. En effet, parmi les innovations artistiques à Florence dès le début $\mathrm{du} \mathrm{XV}^{\mathrm{e}}$ siècle, la sculpture en terre cuite et plus particulièrement en terre cuite émaillée, «inventée » par Luca della Robbia probablement dans les années 1430 et poursuivie par sa famille, joue un rôle majeur. L'intérêt porté à ces œuvres se diffuse rapidement de la Toscane vers l'ensemble de l'Italie d'abord (Venise, Naples...), puis de l'autre côté des Alpes à partir des dernières décennies du $\mathrm{XV}^{\mathrm{e}}$ et surtout au début du $\mathrm{XVI}^{\mathrm{e}}$ siècle. Les robbiesques s'inscrivent parfaitement dans ce goût pour la sculpture italienne qui se diffuse en France dès la fin du XV siècle jusqu'à Fécamp, Dol de Bretagne, Folleville (Picardie) ou Le Mans. Dans le Sud de la France, les liens avec la Toscane passèrent certainement par le roi René d'Anjou, comte de Provence. Jacopo de'Pazzi, l'un de ses conseillers, commanda après 1466 un gigantesque blason en terre cuite émaillée, aux armes de René d'Anjou, pour sa villa de Monthugi, à Fiesole. Un blason similaire ornait le palais comtal du roi René à Aix ${ }^{11}$, que Pierre-Joseph de Haitze ${ }^{12}$ décrit comme « un grand bassin de terre cuite, bordé de plusieurs fruits avec leurs couleurs naturelles aussi vives que s'ils venaient d'être cueillis ». De plus, deux médaillons en terre cuite émaillée représentant des figures, aujourd'hui très abîmées, ornent la maison du chapitre à $\mathrm{Aix}^{13}$. Il faut attendre la deuxième décennie du XVI ${ }^{\mathrm{e}}$ siècle pour trouver une autre commande, à Marseille cette fois, celle d'une imposante « mise au tombeau » en terre cuite émaillée blanche, probablement une commande de la famille Séguier pour sa chapelle de la Vieille Major ${ }^{14}$.

À la suite de nos recherches, il faut donc désormais y ajouter d'autres œuvres ; cependant, étant donné le caractère extrêmement parcellaire des douze fragments, aucune scène n'est immédiatement identifiable et nous ne pouvons 
qu'émettre des hypothèses en nous fondant sur des indices stylistiques, typologiques, des caractéristiques matérielles et des informations glanées dans les archives.

\section{Iconographie et provenance}

L'analyse scientifique isole le fragment à oves (fr. 8, fig. 4) et lui assigne une date un peu postérieure, après 1520. Plus que d'un élément de retable ou de tondo religieux, son motif de coquille bordée d'oves et ses dimensions ${ }^{15}$ le rapprochent de ceux de tondi florentins contenant des armoiries, un exemple analogue étant le tondo au blason de la famille Del Monte appartenant à la collection Contini Bonacossi (Florence, Galerie des Offices), de datation similaire, présentant audessus des oves une couronne extérieure de fruits, fleurs et feuillages ${ }^{16}$.

Pierre Trabaud (1883) évoque par ouï-dire, et sans la mettre en relation avec un élément conservé, « une guirlande de fruits et de fleurs de diverses couleurs devant servir, selon la mode florentine, de cadre arrondi, tondo, arco, à quelque important sujet».J.-A. Mortreuil (1859) est plus précis sur ce dernier point : «On voyait autrefois sur la porte de l'ancienne église de l'Observance les armoiries d'Honoré de Savoie, comte de Tende, grand sénéchal de 1566 à 1572, entourées d'une guirlande de fruits et de feuillages en terre cuite peinte au naturel. C'était sans doute une terra invitriata, par application des procédés italiens, une véritable faïence émaillée. » Ce passage est confirmé (ou reproduit ?) par Meynier (1866) : «Sur la porte de l'Observance on voyait les armoiries d'Honoré de Savoie. Elles étaient en terre cuite et sortaient probablement des ateliers du sieur Philippe Boyer, maître faïencier, voisin du couvent ${ }^{17}$. » J.-A. Mortreuil fait sans doute appel à une mémoire remontant au plus tard à la première moitié du $\mathrm{XVIII}^{\mathrm{e}}$ siècle et à la façade de la première église du XVI ${ }^{\mathrm{e}}$ siècle, souvenir qui a échappé à Joseph Marchand ${ }^{18}$, peintre local.

La période pendant laquelle Honoré de Savoie est gouverneur et grand sénéchal de Provence semble tardive pour la datation issue de l'analyse scientifique. Les armoiries auraient pu être celles de son père, Claude de Savoie, gouverneur de Provence de 1525 à $1566^{19}$. Ce dernier confirmait le 27 octobre 1531 l'autorisation accordée au couvent de

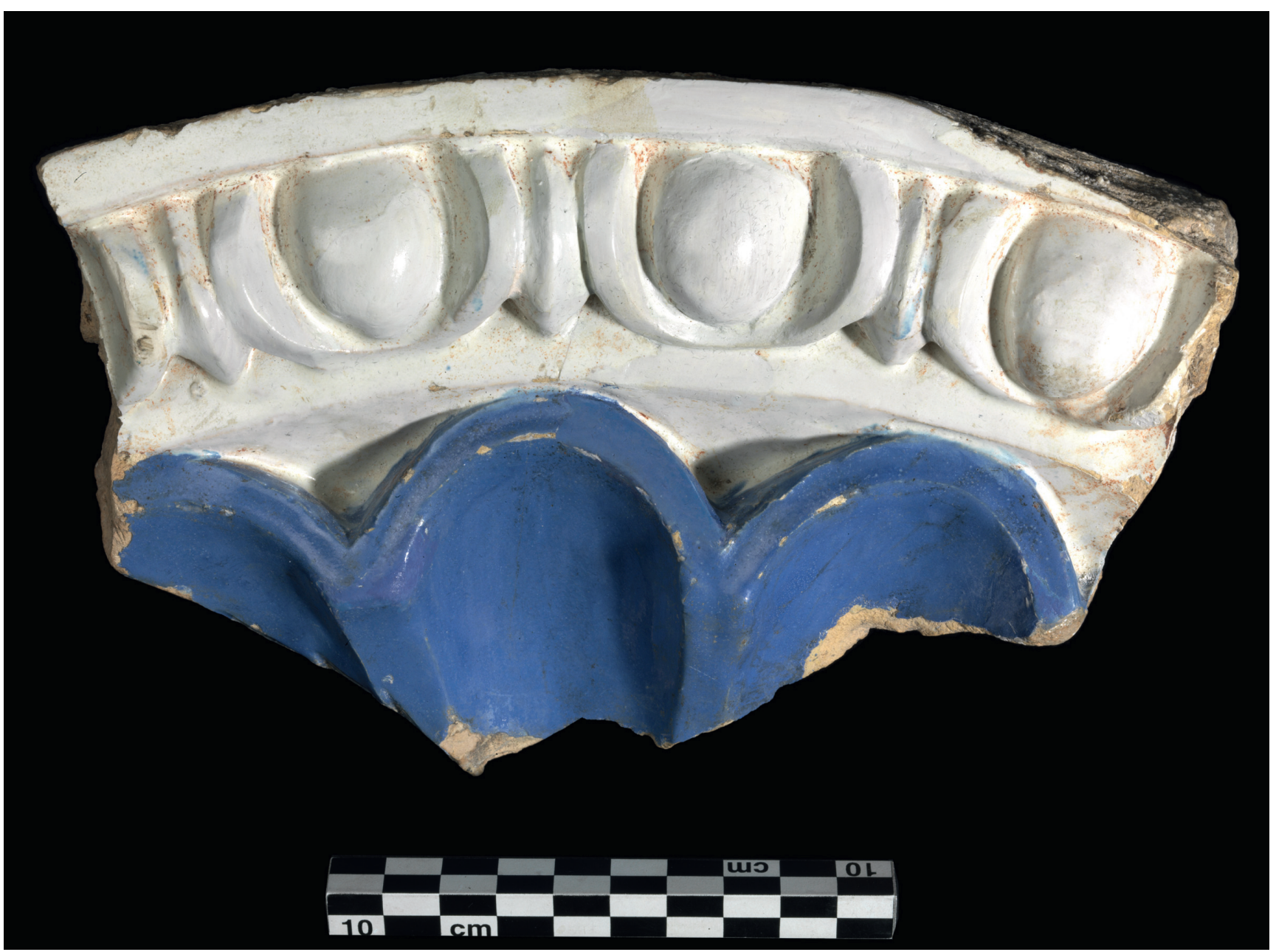

Fig. 4. Fragment de bordure à oves provenant vraisemblablement d'un tondo armorié, musée d'Histoire de Marseille. @ C2RMF/Anne Maigret. 


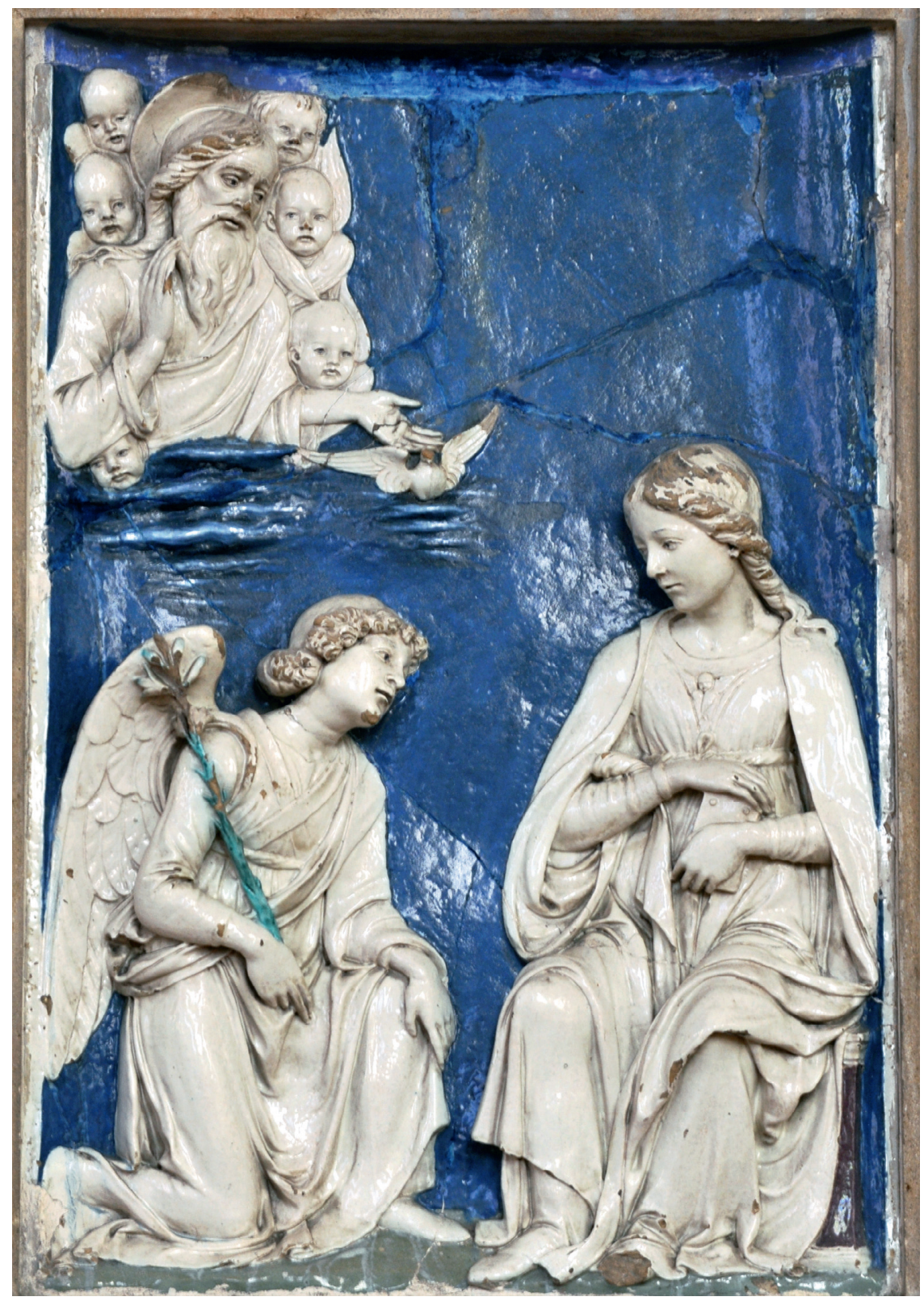

Fig. 5. Andrea Della Robbia, L'Annonciation, église de Saint-Martin-au-Laert. (C) Carl Peterolff. prendre gratuitement de l'eau aux fontaines de la ville ${ }^{20}$. Cette libéralité insigne serait-elle l'indice d'un lien particulier avec le couvent, signalé par le blason du bienfaiteur sur la façade?

L'enquête est plus compliquée pour les onze autres fragments. Repartons de la mention de Joseph Marchand, citée par Pierre Trabaud en 1883, d'un «bas-relief en faïence dans l'ancienne église de l'Observance ». Trabaud se réfère à des « témoins oculaires » qui lui « ont affirmé que ces morceaux étaient, il y a cinquante ans encore assez nombreux pour recomposer d'une manière reconnaissable les tableaux brisés ${ }^{21}$ ». Une première recherche dans les archives centrée sur cette église permet de relever quelques mentions qui pourraient être en rapport avec nos fragments. Plusieurs églises se sont succédé sur le site donné en 1452 aux frères mineurs de l'Étroite Observance près de l'anse de l'Ourse. La première est inaugurée en 1542, quelques décennies après le début de sa construction $^{22}$. Les archives y indiquent une chapelle de l'Annonciation, sépulture des «Florentins ${ }^{23}$ ». A. Rossini [Antoine Ronzen] donne en 1517 le prix-fait d'un «banc et espalier » pour une chapelle de l'église de l'Observance contigüe à la sacristie appartenant à Cosme Botegarii, marchand florentin ${ }^{24}$. Est-ce la même chapelle et contenait-elle une Annonciation robbiesque?

L'église du XVI ${ }^{\mathrm{e}}$ siècle tombe en ruine. La nouvelle est bénite par Mgr de Belsunce en 1746. L'entrepreneur Raymond y pose en 1763 la vitre de « la chapelle de faillance ${ }^{25} »$. Cette appellation semble caractériser une chapelle où ont été transférés des Della Robbia. Les moines peinent à financer la fin 
des travaux et doivent vendre en 1771 les restes de l'ancienne église à l'exception de la chaire, des cloches, des armoiries et épitaphes, du retable et de l'autel de la chapelle de l'Annonciation (contenant l'Annonciation robbiesque ?). L'église du XVIII ${ }^{\mathrm{e}}$ siècle, vendue aux enchères en 1792 , est revendue en 1817 par la veuve de l'acquéreur : «La venderesse se réserve la faculté de faire enlever à ses fraix et risques le tableau en fayence incrusté dans la nef à levant de l'Eglise ${ }^{26}$. »

Quels fragments pourraient provenir d'une Annonciation? Un seul représente un personnage féminin (fr. 4). Un autre (fr. 12) pourrait correspondre aux bras croisés d'un ange. Le fr. 3, représentant un drapé et le montant d'un siège, trouve une correspondance évidente dans une Annonciation des Della Robbia (fig. 5) conservée dans l'église de Saint-Martin-auLaert $^{27}$, près de Saint-Omer, mais aussi dans l'un des éléments de la prédelle du retable du Couronnement de la Vierge, Résurrection et saints de San Bernardino à l'Aquila ${ }^{28}$, d'un autre élément de la prédelle de la Vierge et l'Enfant entre les saints Louis, Catherine d'Alexandrie, Marie-Madeleine et Jérôme de la chapelle Rocca, à Gradara ${ }^{29}$. Les fr. 4 et 3 seraient donc deux parties d'un même personnage, une Vierge, les proportions étant cohérentes avec ce sujet. La présence d'une terrasse parme sur le fr. 3 conduit à s'intéresser au fr. 2, qui comporte une terrasse de même couleur (quoique la découpe de cette base ne s'insère pas dans le fr. 3 ). Il représente la partie inférieure du corps d'un ange (identifiable par les cordelettes à pompon et les rubans frangés flottant autour de la taille, en parfaite cohérence avec deux anges de la collection ThyssenBornemisza $\left.{ }^{30}\right)$. Mais il semble cependant difficile de rapprocher de cet élément le fr. 12 aux bras croisés : la lacune entre le bas du personnage et ce fragment est trop importante, et la position même du corps de l'ange empêche tout raccord au torse. On serait donc en présence de deux fragments d'anges, un torse (fr. 12) et la partie inférieure d'un corps (fr. 2), chacun appartenant à une scène différente, et l'un seulement (le bas du corps sur terrasse parme) à une Annonciation.

Nous n'avons pas envisagé un raccord entre les fragments à terrasse parme et ceux à base verte (fr. 1, 6, 7), car nous avons constaté que les retables des Della Robbia étaient le plus souvent conçus avec des bases monochromes, sauf lorsqu'ils sont composés de plusieurs panneaux séparés par des éléments architecturés.

Des trois éléments (fr. 1, 6, 7) sur terrasse verte, aucun ne peut être assimilé à un personnage d'une Annonciation. Outre le religieux (fr. 7), nous avons à notre disposition un personnage agenouillé (fr. 1) et un fragment de drapé (fr. 6), deux éléments beaucoup trop lacunaires pour les identifier précisément.

Aurions-nous plus de chance avec les hypothèses de Pierre Trabaud à partir des éléments 2, 5, 7, 10, 12 ? Il y voyait une Descente de croix ou un Couronnement de la Vierge. Nous ne savons pas sur quels critères il fondait son assertion : la mémoire locale ou des pièces plus grandes ? Outre l'impossibilité de faire coexister des bases de couleur différente - ce qui exclut soit le fr. 2, soit le fr. 7 - les quatre fragments restants ne sont pas suffisamment parlants. Cependant, les parties de ciel avec des rayons d'or et des flèches trouvent leur parallèle dans des scènes présentant des personnages divins, le soleil ou le christogramme ${ }^{31}$. La direction des rayons vers le haut du ciel et le négatif d'une ou de deux têtes de chérubin plaideraient en faveur de cette lecture. Dans le cas d'un Couronnement de la Vierge, la partie du corps de saint Jean-Baptiste (fr. 9) pourrait y trouver sa place, ainsi que le torse d'ange aux bras croisés (fr. 12).

Les hypothèses sont nombreuses et les inconnues plus encore : comment dans ces conditions, envisager la présentation de ces éléments dans les salles du musée ? Jusqu'à présent, seul le fr. 7, le plus grand, a été montré au public (fig. 6) ${ }^{32}$. D'autres musées possédant des œuvres des Della Robbia fragmentaires ont été confrontés à de telles questions, notamment le musée Sandelin à Saint-Omer. Il expose en effet ce qui reste de la première ouvre robbiesque (en France) documentée correspondant au Tombeau que Guillaume Fillastre $^{33}$, abbé de Saint-Bertin et chef du conseil privé de Philippe Le Bon, duc de Bourgogne, commanda à Andrea della Robbia. Il n'en subsiste plus aujourd'hui que quelques

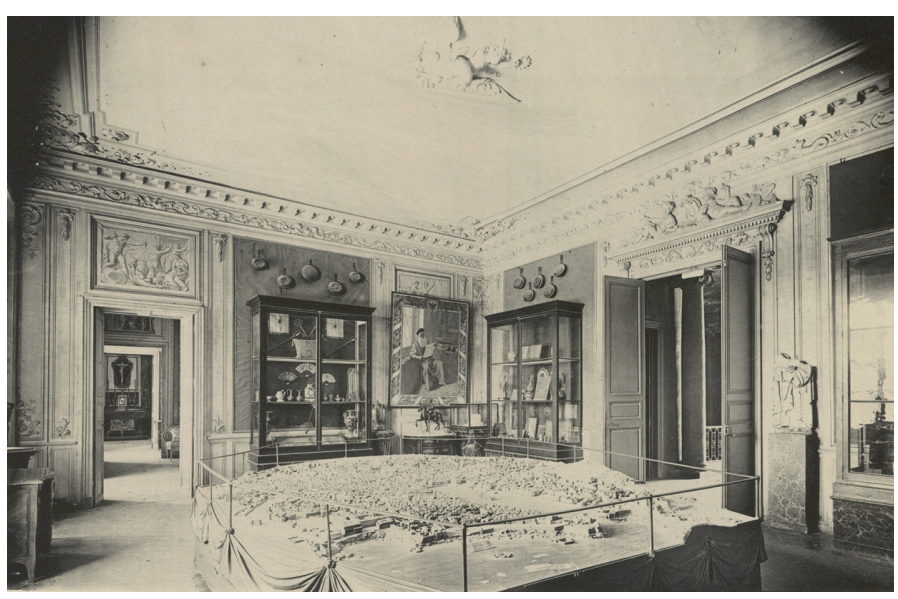

Fig. 6. Le « religieux » fragment 7, exposé au musée Borély au début du Xx ${ }^{\mathrm{e}}$ siècle. (C) Musée d'Histoire de Marseille/S. Aillaud.

reliefs ${ }^{34}$ d'où se détache une épitaphe fragmentaire avec un impressionnant squelette drapé (fig. 7). Trois autres fragments sont présentés (une Annonciation, une Cène, Jérémie), mais ils ont l'avantage, par rapport aux éléments marseillais, d'être individuellement et directement lisibles par le public. Exposer des œuvres fragmentaires est encore une gageure pour un musée des beaux-arts ${ }^{35}$, mais paraît plus naturel dans un musée d'histoire et d'archéologie. Le nouveau parcours du musée d'Histoire de Marseille propose trois solutions différentes pour des fragments de décor religieux d'une même église, Saint-Martin, détruite au XIX ${ }^{\mathrm{e}}$ siècle. Les éléments en bois polychromé d'un orgue baroque sont visibles dans une alcôve ; des têtes de chérubins en marbre sont posées sur des 
platines fixées au mur ; enfin les vestiges lapidaires sont disposés sur des racks. En s'inspirant de ces trois modalités, le plus grand nombre des fragments de céramique seraient alors visibles. On pourrait imaginer deux socles différents et non jointifs afin d'y faire reposer les fragments sur terrasse, l'un pour les terrasses vertes et l'autre pour les bases parme. Les éléments quadrangulaires bleus (fr. 5 et 10), ainsi que le bord (fr. 11) pourraient être disposés en arrière-plan sur le mur, laissant apparaître une lacune arbitraire entre eux. Les trois vestiges de corps (fr. 4, 9, 12) pourraient être présentés sur un plan incliné dans une vitrine. Ainsi seraient rendues perceptibles tant la difficulté de reconstituer une scène que les différentes hypothèses.

Le choix d'exposer l'ensemble des fragments est soustendu par l'espoir de voir réapparaître d'autres éléments permettant petit à petit de reconstituer les scènes : entre la vente de l'église en 1817 et la présentation de ces éléments au XXI ${ }^{\mathrm{e}}$ siècle, la dispersion du ou des retables et la résurgence de certains éléments entre 1864 et 1882 laissent augurer un phénomène comparable. Nous en voulons pour preuve un cas analogue : la Marie-Madeleine (Berne, musée d'Histoire) était présentée incomplète depuis 1925 lorsque des vestiges de terre cuite furent donnés par le pasteur au musée. De nouvelles fouilles en 1976 ont livré quelques fragments supplémentaires qui ont permis d'enrichir la représentation de la sainte, sans lui rendre tout son volume, comme le montre la photo dans le catalogue de l'exposition « Iconoclasme » à Berne $^{36}$.

\section{Conclusion}

L'étude historique et scientifique conforte le statut de ces fragments robbiesques : la «faïence » de Marseille est issue des recherches menées par les maîtres florentins. Elle apporte aussi des éclairages sur l'histoire urbaine, en redonnant vie à l'église de l'Observance et à son décor. Les éléments de sculpture en céramique qui ont subsisté malgré les déplacements et les destructions constituent un jalon important dans l'histoire de la production glaçurée importée à Marseille. Outre la Mise au tombeau de la Vieille Major, conservée sur place et le Christ en croix vêtu d'une robe disparu avec une partie de l'église des Accoules, on doit tenir compte des fragments du musée d'Histoire de Marseille qui proviennent vraisemblablement du couvent de l'Observance.
Les pistes ouvertes devront être encore explorées pour une meilleure appréciation de la diffusion d'une technique novatrice et du rôle de passeurs des marchands italiens.

\section{Remerciements :}

Nous tenons à remercier chaleureusement pour leur aide et leurs conseils Régis Bertrand, Brigitte Bourgeois, JeanRené Gaborit, Marie Lionnet, Marie-Lys Marguerite, Anne Philippon, Georges Reynaud, Francine Valette, ainsi que toute l'équipe AGLAE.

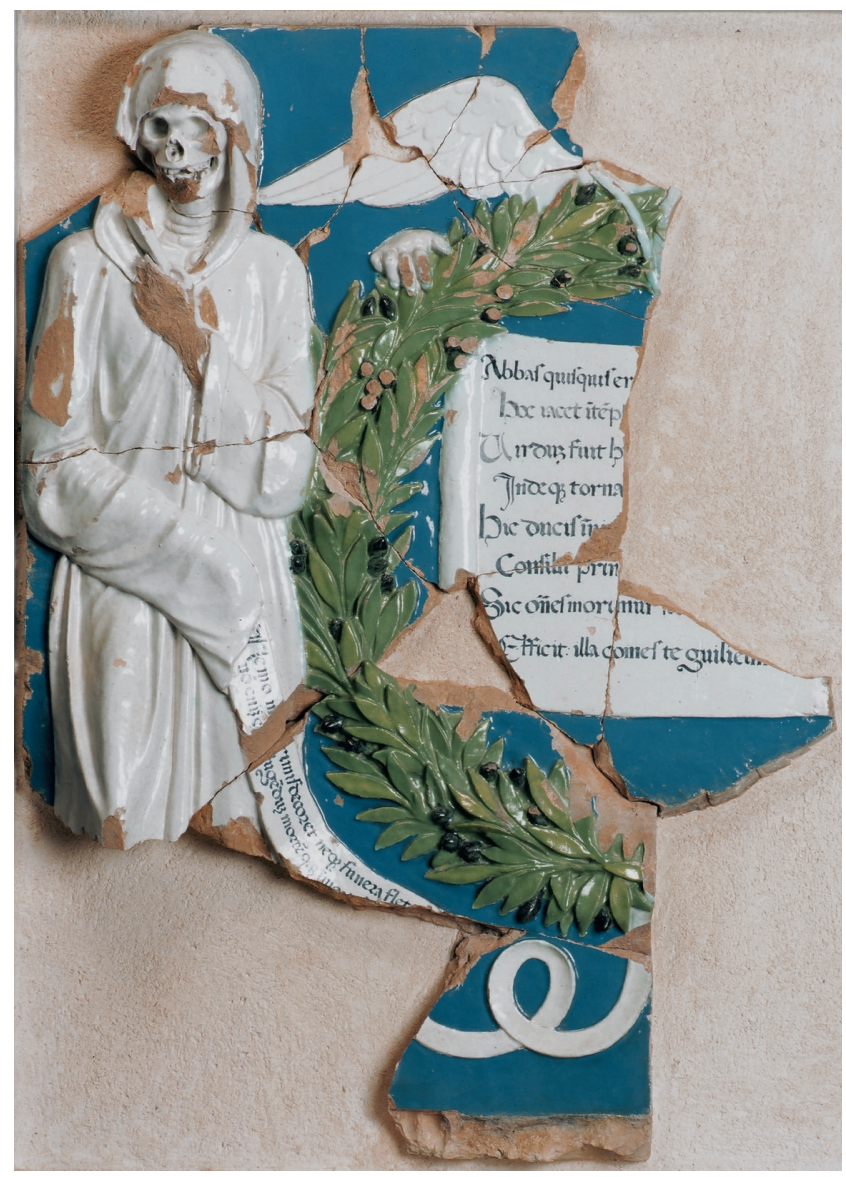

Fig. 7. Andrea Della Robbia, La Mort et l'épitaphe, vers 1469-1470, terre cuite émaillée (inv. 2795 ter), Saint-Omer, musée de l'hôtel Sandelin. (C) Philippe Beurtheret.

\section{Notes}

1. Voir B. Beillard, A. Bouquillon, B. Mottin, 2001, p.150-156.

2. Inventoriées à l'occasion de leur restauration (2014.0.1 à 2014.0.12 et désignées ici par fr. 1 à fr. 12).

3. Historien, archéologue, $2^{\mathrm{e}}$ directeur du musée d'Archéologie méditerranéenne de Marseille (1895-1928).
4. Hippolyte Augier (1830-1889) est surtout connu pour avoir réalisé 79 maquettes de sites archéologiques et monuments du Midi de la France dont 25 concernent Marseille. Morel-Deledalle M., 2004, p. 36-42. Il inscrivait ses dons et achats dans des carnets. La bibliothèque du MHM en possède un mais aucune mention n'est faite des éléments qui nous intéressent ici.

5. P. Trabaud, 1883, 1 et 2 (publications identiques) ; le 7 décembre 1882, il donne lecture de son article à l'Académie de Marseille.

6. Il reproduit p. 17 le dessin par Michel de Léon en $1778 \mathrm{n}^{\circ} 61$ (Voyage pittoresque ...). Cet auteur en a indiqué l'emplacement «au fond de la nef sous les orgues » sur l'autel de la famille de Vento (Taphologe ...). Le mariage de Louis de Vento avec Elisabeth Meillori, d'une famille illustre de Lucques, dont le fils naquit en 1519 expliquerait cette représentation du Volto Santo lucquois. 
7. Remarquons que ces deux morceaux ne sont pas cités dans l'inventaire de 1895 .

8. L'abbé Dassy lui a-t-il confié l'un des débris du Christ en faïence des Accoules qu'il avait récupérés lors des démolitions engendrées par la construction du Calvaire à l'emplacement des restes de l'église, de même qu'il en a communiqués à Mortreuil pour les analyser visuellement (Mortreuil, 1859)?

9. Zucchiatti et Bouquillon, 2011.

10. Zucchiatti et Bouquillon, 2011.

11. Détruit en 1776-1786.

12. P.-J. de Haitze, 1679, in J.-J. Gloton 1979 , p. 3.1 . 331.

13. J.-J. Gloton, R. Guild, 1989, p. 325-

14. B. Beillard, A. Bouquillon, B. Mottin, 2001, p. 150-156 ; J.-R. Gaborit, 2002, p.122-123.

15. Le diamètre d'un tel tondo est de 0,50 à $0,55 \mathrm{~m}$ pour la bordure d'oves, soit, avec la guirlande extérieure un peu moins de 1 mètre.

16. G. Gentilini, 1992, vol. II, ill. p. 307.

17. J.-A. Mortreuil, 1859, p. 272 ;

Meynier, 1866, p. 543.

18. Portefeuille du peintre Joseph-

Martin Marchand, 1794-1805, Archives des Bouches-du-Rhône (AD 13), 50 Fi, image 127 sur archives13.fr : " Il y avait dans l'ancienne église de l'Observance un basrelief en fayence, un autre qui est dans l'église de la Major, et un Christ couvert d'une tunique encore en fayence dans l'église des Accoules. Ces ouvrages paraissaient être faits par les Della Robbia : Florentins qui étaient venus en France sous François Ier. (...) Il est vraisemblable que cette famille d'artistes vint débarquer à Marseille. » Trabaud a consulté le document alors qu'il appartenait à l'abbé Louche.

19. Nous excluons le père de Claude, René de Savoie, nommé gouverneur et grand sénéchal de Provence par son neveu François I ${ }^{\mathrm{er}}$, dit « le bâtard de Savoie », dont les armoiries sont reconnaissables à la barre indiquant cet état.

20. AD 13, fonds de l'Observance $37 \mathrm{H} 7$.

21. P. Trabaud 1, p. 16, ajoute : «dans les caves d'une chapelle Saint-Sauveur place de Lenche ... des fragments de terre cuite et des têtes de saints dont les petits mauvais sujets jouaient comme des boules. »

22. Les Pénitents noirs de saint JeanBaptiste y établissent leur chapelle en 1521. L'église, d'abord sous le vocable de saint François, passe sous celui de saint Louis par lettre de Clément VII en 1531, la consécration à saint Louis intervient 11 ans plus tard. Belsunce, 1751, p. 164-165.

23. AD 13, fonds de l'Observance 37 H 3 p.11.

24. Albanès, 1884, p. 283 et 292-293.

25. Compte des journées et fourniture faittes au couvent de l'Observance, le 17 septembre 1763 : «garnir la vitre de la chapelle de faillance ", acquitté par Jean Étienne Raymond le 4 septembre 1764. AD 13, fonds de l'Observance $37 \mathrm{H} 12$.
26. Reynaud G., 1994, p. 105-109. L'acte de vente ARNAUD/ROUMIEU de 1817 est passé chez Me Borély, AD 13, 357 E 261, acte $n^{\circ} 540$. Nous remercions G. Reynaud de cette précision.

27. Élément du tombeau de Guillaume Fillastre.

28. Gentilini, 1992, vol. I, ill. p. 259

29. Id., vol.I, ill. , p. 210.

30. Radcliffe, Baker, Maek-Gérard, 1992, n 12, p. 100-105, ill. p. 102-103.

31. Soleil sur la Crucifixion de La Verna, christogramme au-dessus du couronnement de la Vierge, couvent Saint Bernardin, Sienne.

32. A. Guérinet (éd.), 1908, pl. 99.

33. En dernier lieu, J.-R. Gaborit 2003, p.50-63.

34. Bormand, Lancestremère, 2004, p. 120 .

35. Faut-il restaurer les ruines ?, 1991 ; M. Polonowski, 2000.

36. Iconoclasme. Vie et mort de l'image médiévale, 2001, ill. 158, p. 324, inv. 16743 , vers $1450,91 \times 31 \mathrm{~cm}$.

\section{Bibliographie}

Albanès abbé, 1884, « Nouveaux documents sur le peintre Antoine Ronzen dit le Vénitien ", Bulletin archéologique du Comité des travaux historiques et scientifiques.

Beillard B., Bouquillon A., Mottin B., 2001, «La déploration sur le Christ mort d'Andrea Della Robbia ", Technè, n $13-14$, p. 150-156.

Belsunce Henri-François-Xavier de, Antiquité de l'Église de Marseille, vol. 3, livre XIV, Marseille 1753-1751.

Bormand M., Lancestremère C., 2004, «Della Robbia : de la difficulté des choix d'intervention ", dans Terres cuites de la Renaissance, Technè, $\mathrm{n}^{\circ}$ 20, Paris, p. 118-126.

Bormand M., Bouquillon A., Doublet C., 2012, «Terres célèbres, terres révélées et terres énigmatiques : terres et ateliers dans la France de la Renaissance », Technè, $\mathrm{n}^{\circ} 36$, Paris, p. 63-71.

Bouquillon A, Bormand M., Zuchiatti A., 2011, Della Robbia. Dieci anni di studiDix ans d'études, Gênes.

Faut-il restaurer les ruines?, 1991, Entretiens du Patrimoine, colloque organisé par la direction du Patrimoine, Caen.

Gaborit J.-R., Bormand M. (dir), 2002 , Les Della Robbia. Sculptures en terre cuite émaillée de la Renaissance italienne, cat. exp. Nice Musée National Message Biblique Marc Chagall 29 juin11 novembre 2002, Sèvres Musée National de Céramique 10 décembre 2002-10 mars 2003, RMN, Paris, 2002, p. $122-123$.

Gaborit J.-R., 2003, « Une œuvre de l'atelier des Della Robbia : le tombeau de Guillaume Fillastre à l'abbaye
Saint-Bertin ", Bulletin de la Société des Antiquaires de France, p. 50-63.

Gentilini, G., 1992, I Della Robbia. La scultura invetriata nel Rinascimento, 2 vol., Milan.

Gloton J.-J., 1979, Renaissance et Baroque à Aix-en-Provence. Recherches sur la culture architecturale dans le midi de la France de la fin du XV $V^{e}$ s. au début du XVIII ${ }^{e}$ s., 2 vol., Rome.

Gloton J.-J., Guild R., 1989, « La cathédrale d'Aix-en-Provence : la façade Renaissance de la maison du Chapitre ", Bulletin monumental 47-4, p. 352-331.

Haitze P-J. de, 1679, Les curiosités les plus remarquables de la ville d'Aix-en-Provence, Aix.

Iconoclasme, vie et mort de l'image médiévale, Dupeux C., Jezler P., Wirth J., 2001, cat. exp. du musée de l'Cuvre Notre-Dame de Strasbourg et du musée d'Histoire de Berne.

Meynier, 1866, "Les anciens chemins de Marseille ", Revue de Marseille et de Provence.

Michel de Léon François, 1778, Voyage pittoresque de Marseille..., Marseille, bibliothèque de l'Alcazar, ms 2101 ; Taphologue de Marseille, ibidem, ms 2102.

Monographie du Palais Borély à Marseille, 1908, Librairie d'Architecture et d'Art décoratif, A. Guérinet (éd.), Paris.

Morel-Deledalle M., 2004, «Hippolyte Augier, un maquettiste au service de l'archéologie », Marseille, p. 36-42.

Mortreuil J.-A., 1859, « Mémoire sur les faïences, verres, émaux, porcelaines (Anciennes industries marseillaises)», Répertoire des travaux de la Société de statistique de Marseille, tome $22^{\mathrm{e}}$ de la $5^{\mathrm{e}}$ série, Marseille.

Polonowski M., 2000, «Le portail disparu de l'église abbatiale de Saint-Pierre de Lagny ", dans Utilis est lapis in structura, Mélanges offerts à L. Pressouyre, CTHS, Paris.

Reynaud G., 1994, «Demeures et propriétés marseillaises de Pierre Puget », dans Cahiers du Comité du Vieux Marseille, $\mathrm{n}^{\circ} 64$.

Radcliffe A, Baker M., Maek-Gérard M., 1992, The Thyssen-Bornemisza Collection. Renaissance and later sculpture, Londres.

Trabaud P. (1), 1883, Les Della Robbia à Marseille, Société Anonyme de l'Imprimerie Marseillaise, Marseille.

Trabaud P. alias Citharis M. T. de (2), 1883, «Les Della Robbia de Marseille ", dans La Provence artistique et pittoresque, $\mathrm{n}^{\circ} 107$, p. 187 et suiv. ; n ${ }^{\circ} 109$, p. 202 et suiv. ; $n^{\circ} 110$, p. 211 et suiv.

Zucchiatti A. et Bouquillon A., 2011, "Les glaçures : atout maître des Della Robbia ", dans Bouquillon A., Bormand M., Zucchiatti A. (eds), Della Robbia, Dix ans d'études. Diecianni di Studi, Sagep, Gênes, p. 27-37. 